\title{
Author Correction: Genetic mapping of cell type specificity for complex traits
}

Kyoko Watanabe (D), Maša Umićević Mirkov, Christiaan A. de Leeuw (D), Martijn P. van den Heuvel(D) \& Danielle Posthuma (1)

Correction to: Nature Communications https://doi.org/10.1038/s41467-019-11181-1, published online 19 July 2019.

The original version of this Article contained an error in Fig. 5 and Supplementary Fig. 12, in which the colours of the bar plots at the top of the heatmaps were incorrectly given, leading to incorrect mapping of the plots to the corresponding dataset based on the dataset legend.

The correct version of Fig. 5 is:

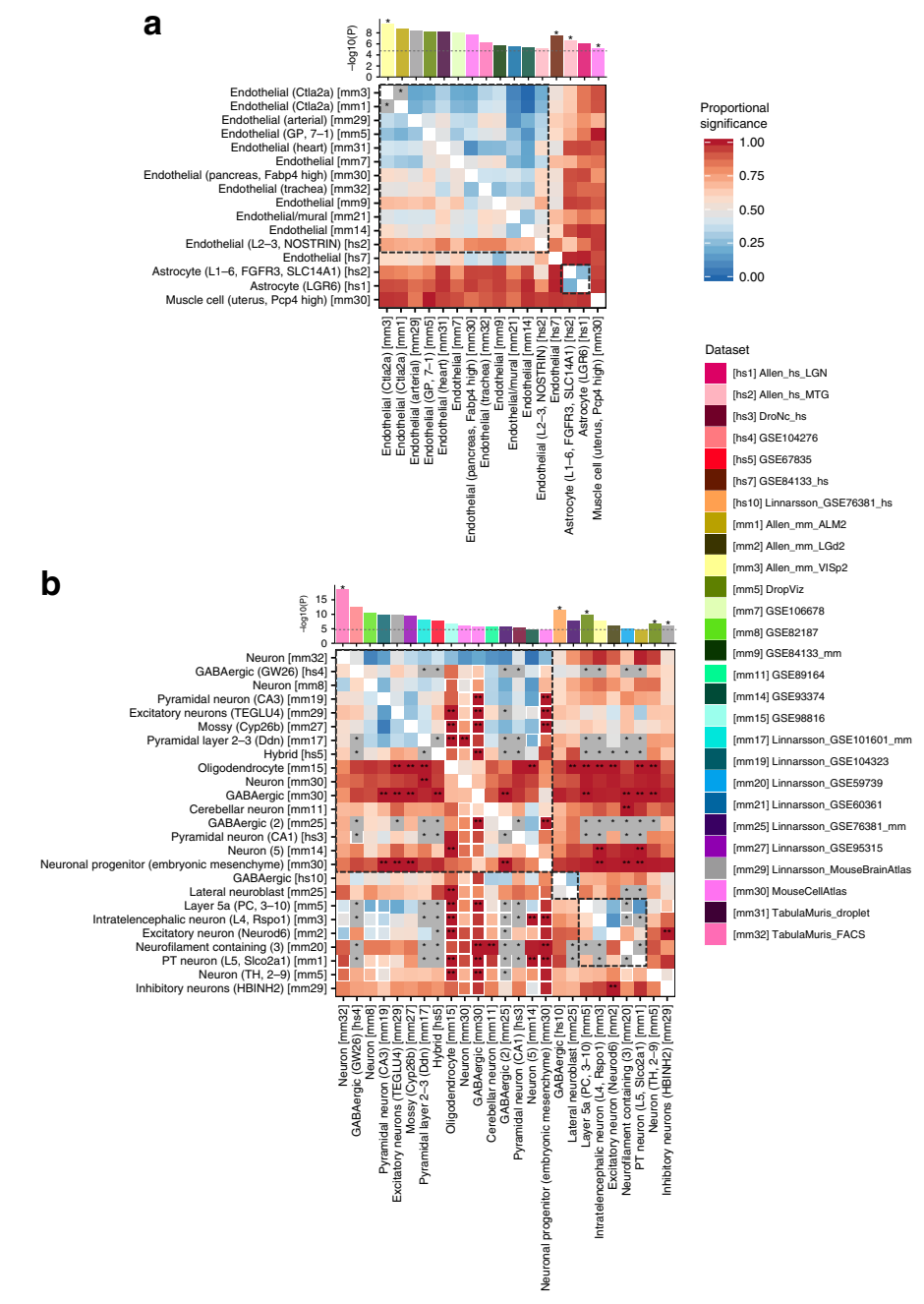


which replaces the previous incorrect version:

\section{a}

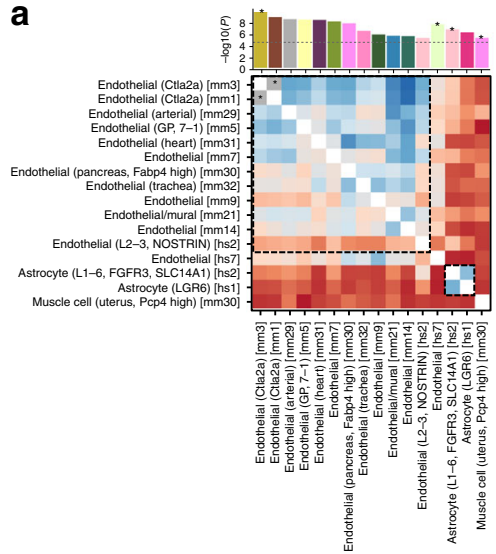

b

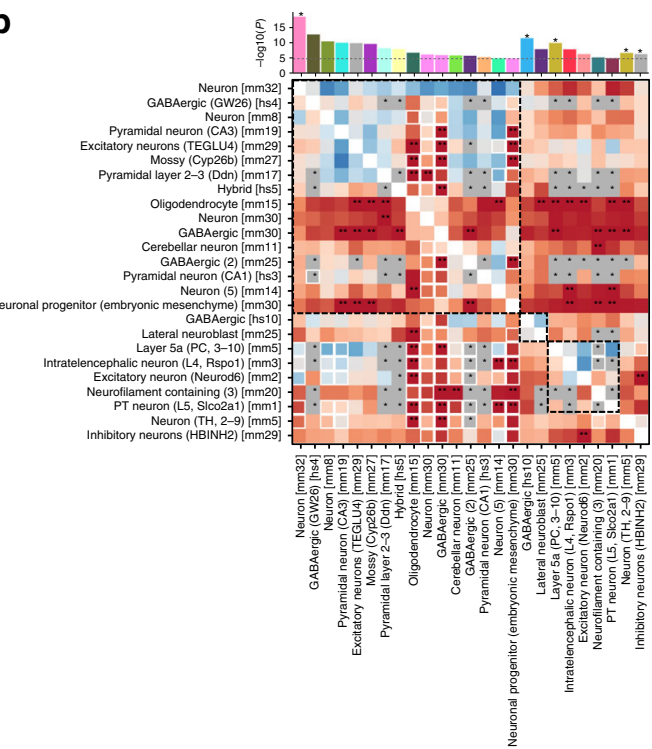

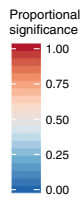

Dataset

[hsi] Allen_hs_LGN

[hs3] Dronc_hs

[hS4] GSE10427

[hs5] GSE67835

[hs10] Linnarsson_GSE76381_hs

[mm1] Allen_mm_ALM2

mm2] Allen_mm_LGd2

[mm5] DropViz

$[\mathrm{mm} 7]$ GSE106678

[mm8] GSE82187

(n)

mm9] GSE84133_m

(m)

[mm15] GSE98816

[mm15] GSE98816

[mm17] Linnarsson_GSE101601_m

[mm20] Linnarsson_GSE59739

SE6036

[mm25]

[mm27] Linnarsson_GSE95315

[mm29] Linnarsson_Mouse

(mm30] MouseCellatlas

[mm31] TabulaMuris_drople

This has been corrected in both the PDF and HTML versions of the Article. 
The correct version of Supplementary Fig. 12 is:

a

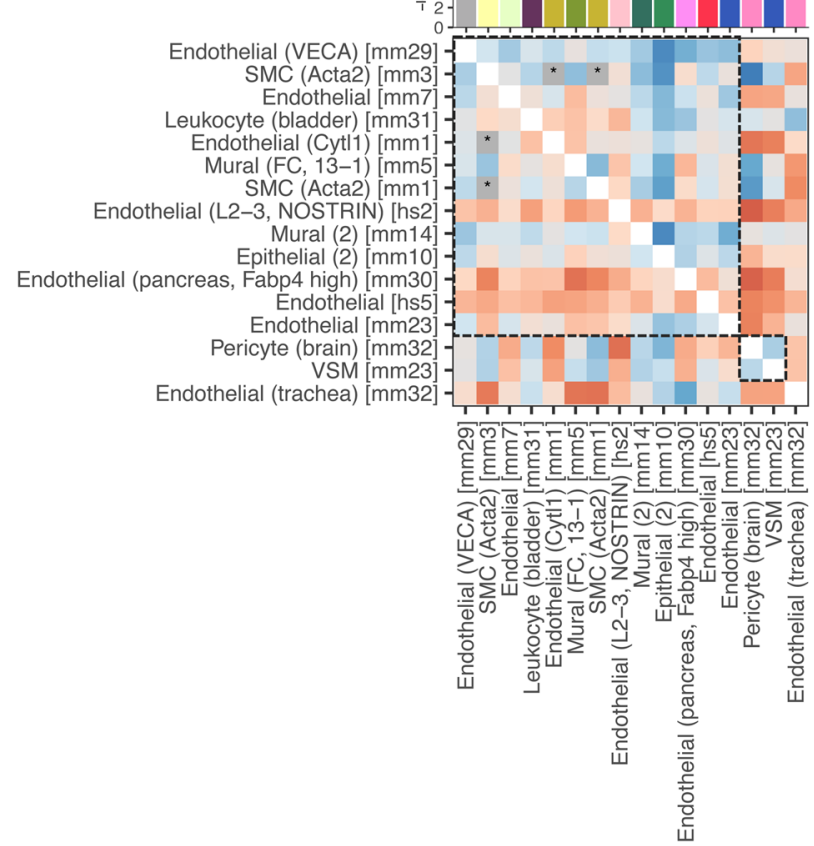

c b

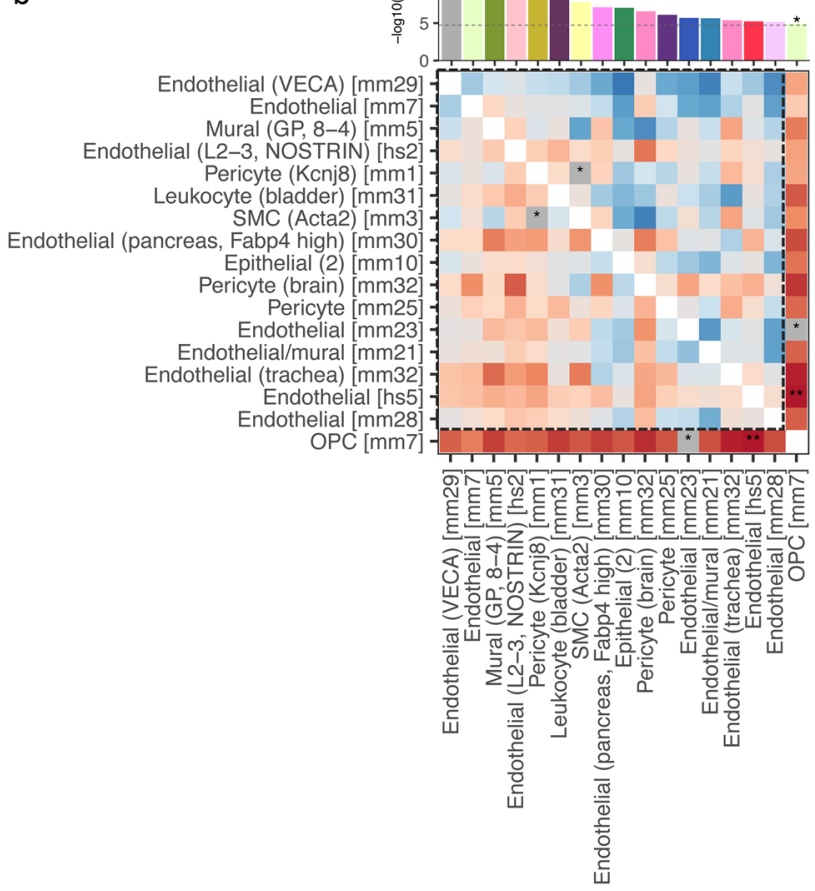

d

Cardiac muscle (heart) [mm31] Cardiac muscle (heart) [mm32] Smooth muscle (heart) [mm31] SMC (Acta2) [mm3] Endothelial (VECA) [mm29] Endothelial [mm7] Mural (TH, 5-14) [mm5]
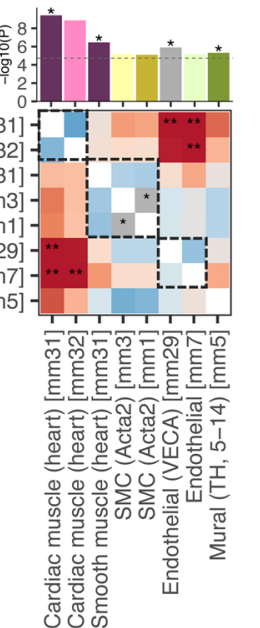
elial (VECA) [mm29]
Endothelial [mm7] Mural (GP, 8-4) [mm5] SMC (Acta2) [mm3 Leukocyte (bladder) [mm31] Epithelial (2) [mm10] Picyte (Kcnj8) [mm1] Mural (2) [mm14] Endothelial [mm28] Endothelial [hs10] Endothelial (trachea) [mm32] Endothelial [mm23] Endothelial (Cyt11) [mm1] VSM [mm23]
Pericyte [mm25] Pericyte (brain) [mm32] Oligodendrocyte (2) [mm7]

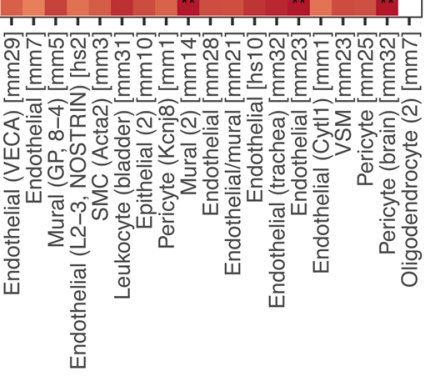

[hs1] Allen_hs_LGN [hs2] Allen_hs_MTG

[hs3] DroNc_hs [hs4] GSE104276 [hs5] GSE67835 [hs6] GSE81547 [hs7] GSE84133_hs [hs10] Linnarsson_GSE76381_hs [hs11] PBMC_10x_68k [mm1] Allen_mm_ALM2 [mm2] Allen_mm_LGd2 [mm3] Allen_mm_VISp2 [mm5] DropViz [mm7] GSE106678 [mm8] GSE82187 [mm9] GSE84133_mm [mm10] GSE87544 [mm11] GSE89164 [mm14] GSE93374 [mm15] GSE98816 [mm17] Linnarsson_GSE101601_mm [mm19] Linnarsson_GSE104323 [mm20] Linnarsson_GSE59739 [mm21] Linnarsson_GSE6036 [mm22] Linnarsson_GSE67602 [mm23] Linnarsson_GSE74672
Proportional

[mm24] Linnarsson_GSE75330 [mm25] Linnarsson_GSE76381_mm [mm27] Linnarsson_GSE95315 [mm28] Linnarsson_GSE95752 [mm29] Linnarsson_MouseBrainAtlas [mm30] MouseCellAtlas

[mm31] TabulaMuris_droplet [mm32] TabulaMuris_FACS significance

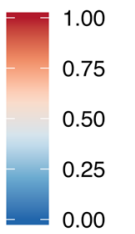



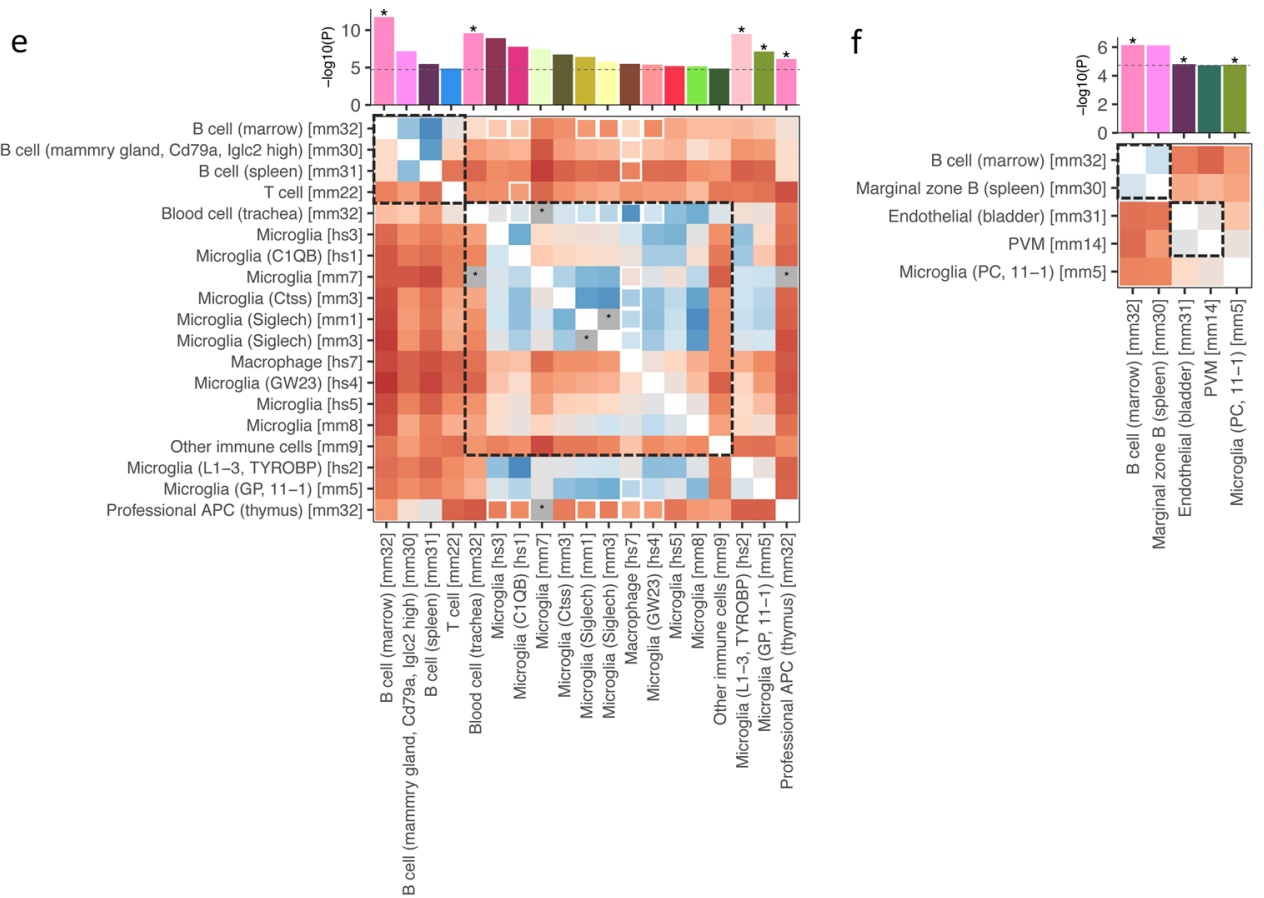

g

[hs1] Allen_hs_LGN [hs2] Allen_hs_MTG [hs3] DroNc_hs [hs4] GSE104276 [hs5] GSE67835 [hs6] GSE81547 [hs7] GSE84133_hs [hs10] Linnarsson_GSE76381_hs [hs11] PBMC_10x_68k

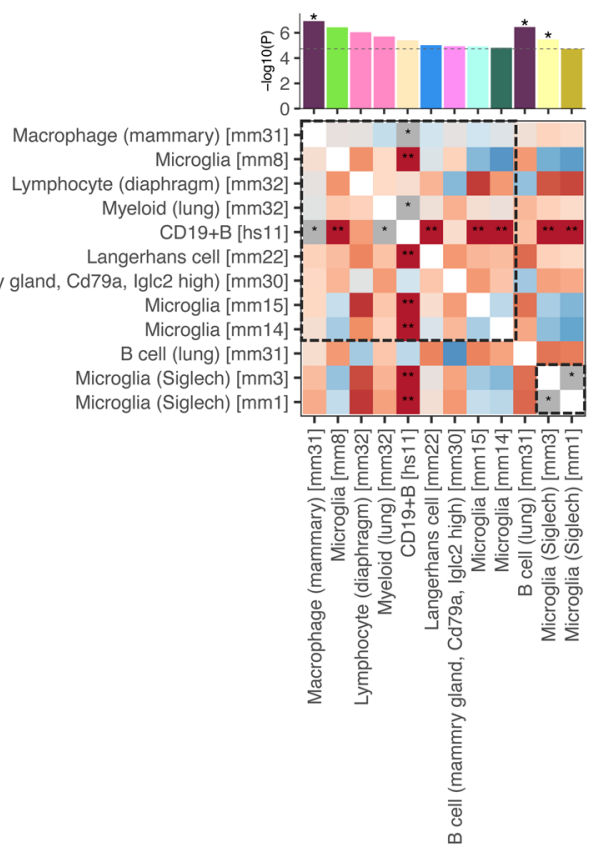

[mm11] GSE89164 [mm14] GSE93374 [mm15] GSE98816 [mm17] Linnarsson_GSE101601_mm [mm19] Linnarsson_GSE104323 [mm20] Linnarsson_GSE59739 [mm21] Linnarsson_GSE60361 [mm22] Linnarsson_GSE67602 [mm23] Linnarsson_GSE74672 h

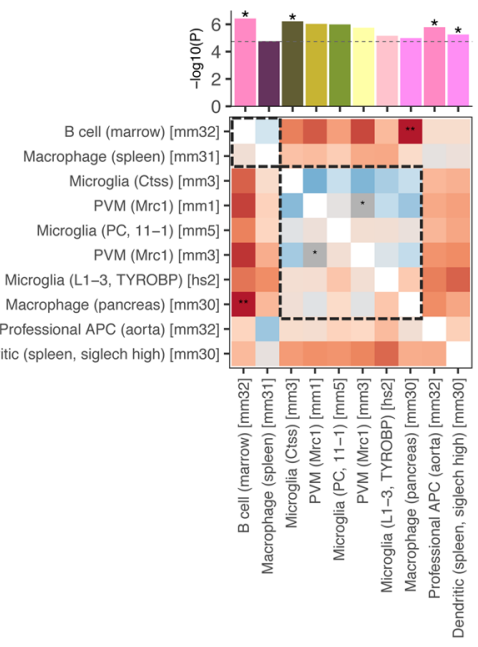

[mm24] Linnarsson_GSE75330 [mm25] Linnarsson_GSE76381_mm

[mm27] Linnarsson_GSE95315 [mm28] Linnarsson_GSE95752 [mm29] Linnarsson_MouseBrainAtlas [mm30] MouseCellatlas

[mm31] TabulaMuris_droplet [mm32] TabulaMuris_FACS
Proportional significance

$=1.00$
0.75
0.50
0.25
$=0.00$


i

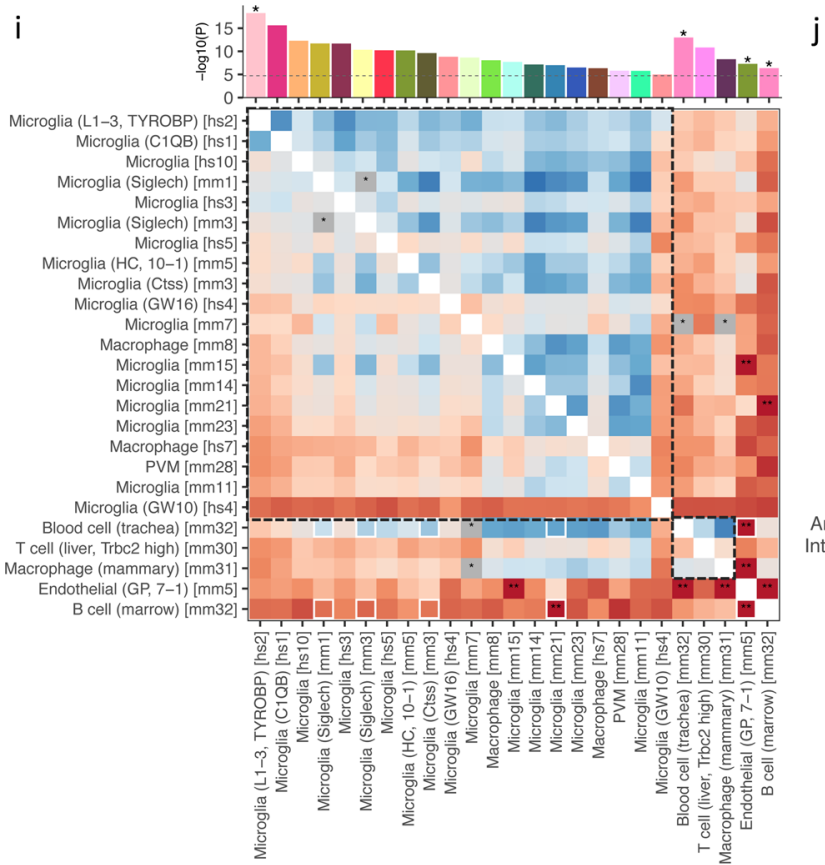

k

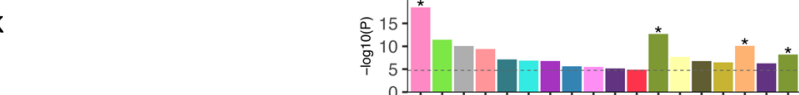

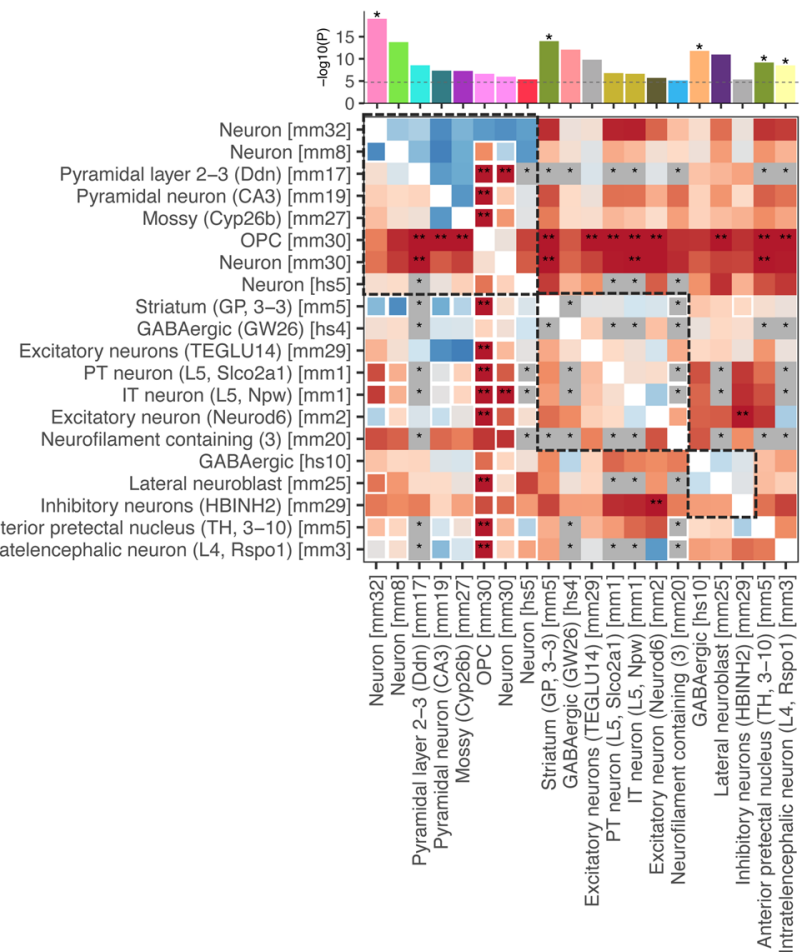

।

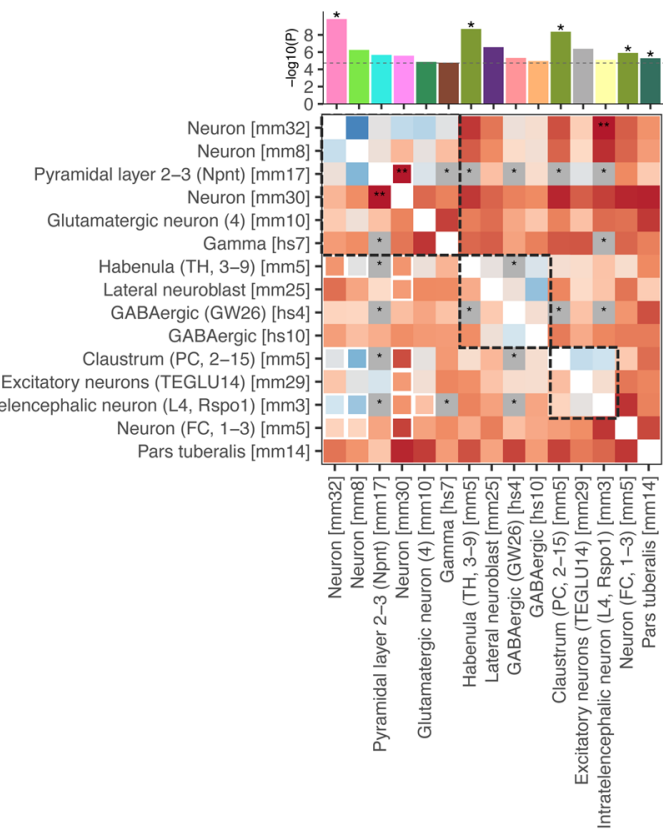

Proportional

significance

[mm24] Linnarsson_GSE75330

[mm25] Linnarsson_GSE76381_mm

[mm27] Linnarsson_GSE95315

[mm28] Linnarsson_GSE95752

[mm29] Linnarsson_MouseBrainAtlas

[mm30] MouseCellAtlas

[mm31] TabulaMuris_droplet

[mm32] TabulaMuris_FACS

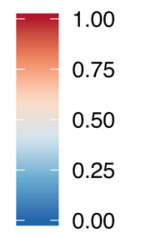


m

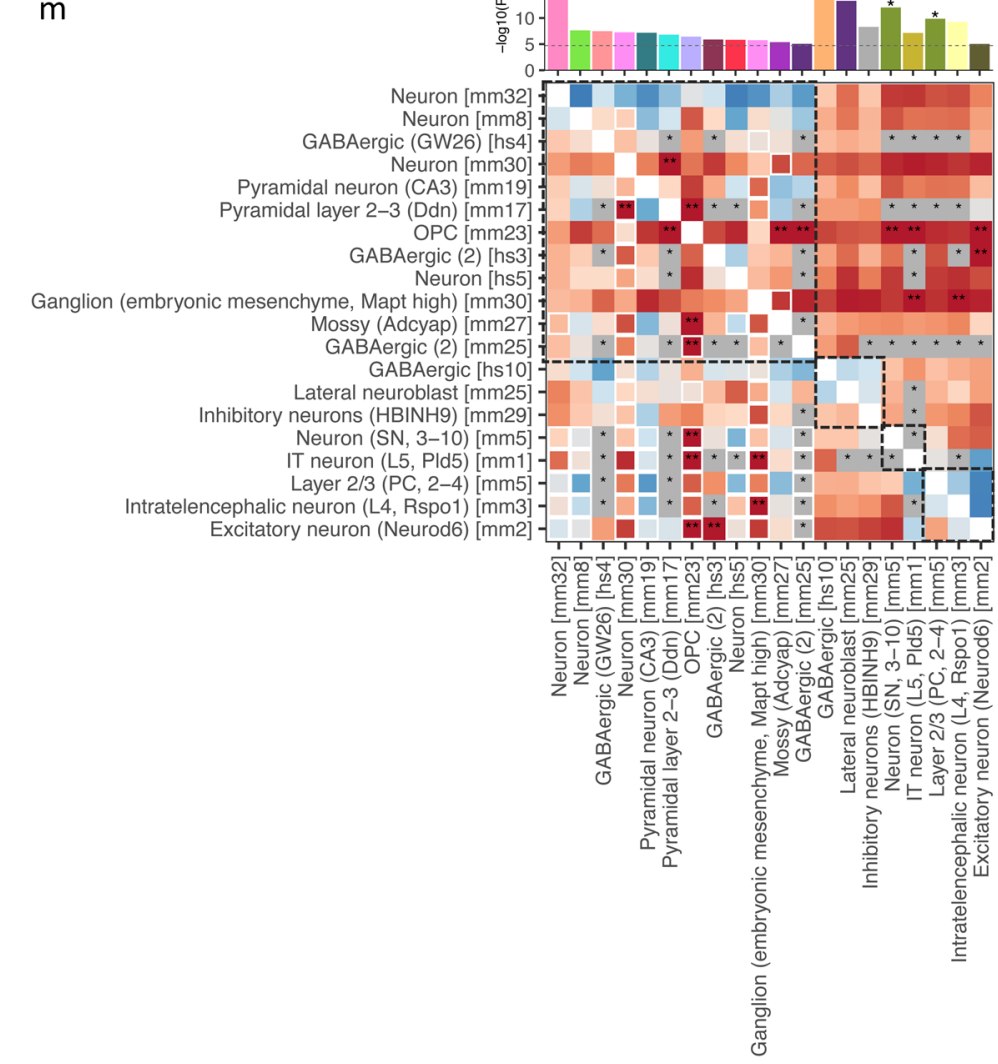

o

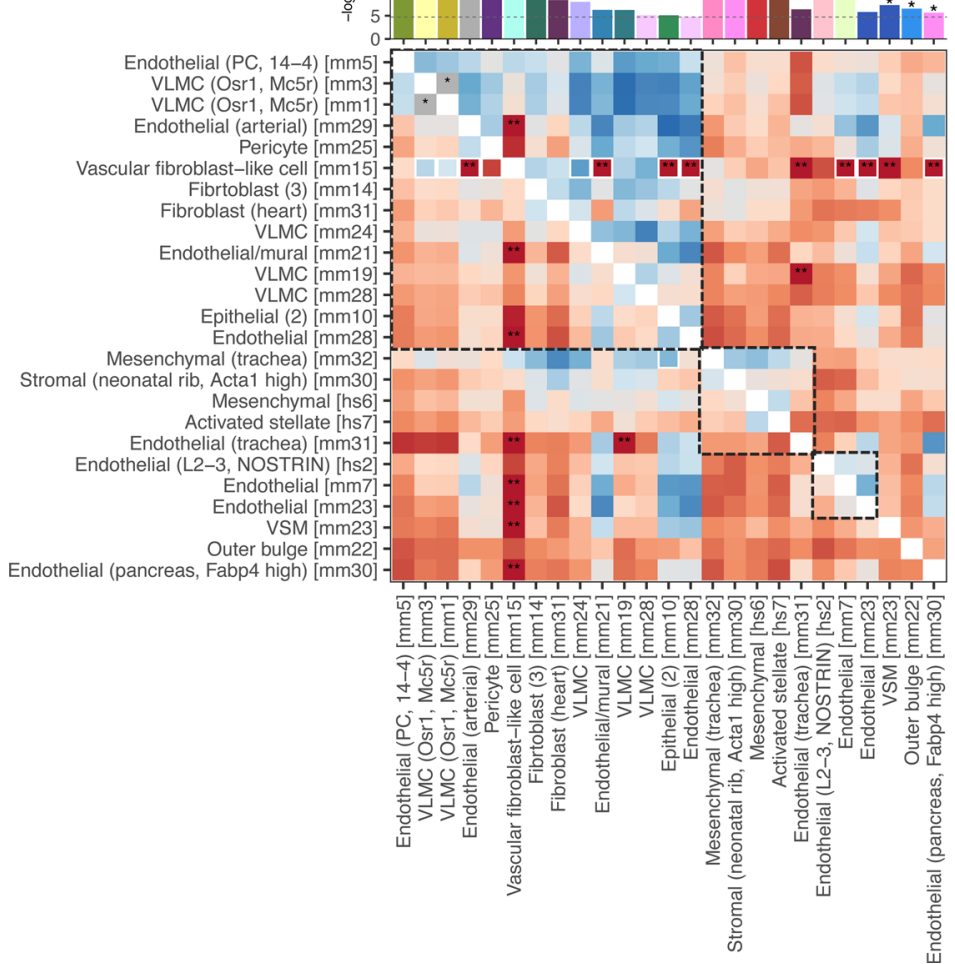

$\mathrm{n}$

Neuron (SN, 4-3) [mm5] Neuron [mm32]

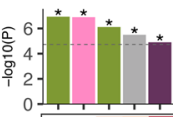

Lateral dorsal thalamic nuclei (TH, 2-6) [mm5] Inhibitory neurons (HBINH6) [mm29]

Leukocyte (bladder) [mm31]

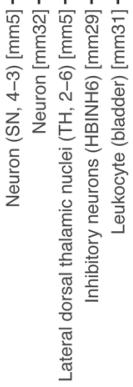

\section{Proportional}

significance

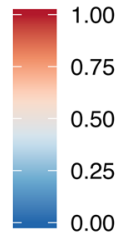

[hs1] Allen_hs_LGN

[hs2] Allen_hs_MTG

[hs3] DroNc_hs

[hs4] GSE104276

[hs5] GSE67835

[hs6] GSE81547

[hs7] GSE84133 hs

[hs10] Linnarsson_GSE76381_hs

[hs11] PBMC_10x_68k

[mm1] Allen_mm_ALM2

[mm2] Allen_mm_LGd2

[mm3] Allen_mm_VISp2

[mm5] DropViz

[mm7] GSE106678

[mm8] GSE82187

[mm9] GSE84133_mm

[mm10] GSE87544

[mm11] GSE89164

[mm14] GSE93374

[mm15] GSE98816

[mm17] Linnarsson_GSE101601_mm

[mm19] Linnarsson_GSE104323

[mm20] Linnarsson_GSE59739

[mm21] Linnarsson GSE60361

[mm22] Linnarsson_GSE67602

[mm23] Linnarsson GSE74672

[mm24] Linnarsson_GSE75330

[mm25] Linnarsson_GSE76381_mm

[mm27] Linnarsson_GSE95315

[mm28] Linnarsson_GSE95752

[mm29] Linnarsson_MouseBrainAtlas

[mm30] MouseCellAtlas

[mm31] TabulaMuris_droplet

[mm32] TabulaMuris_FACS 
which replaces the previous incorrect version:.

a

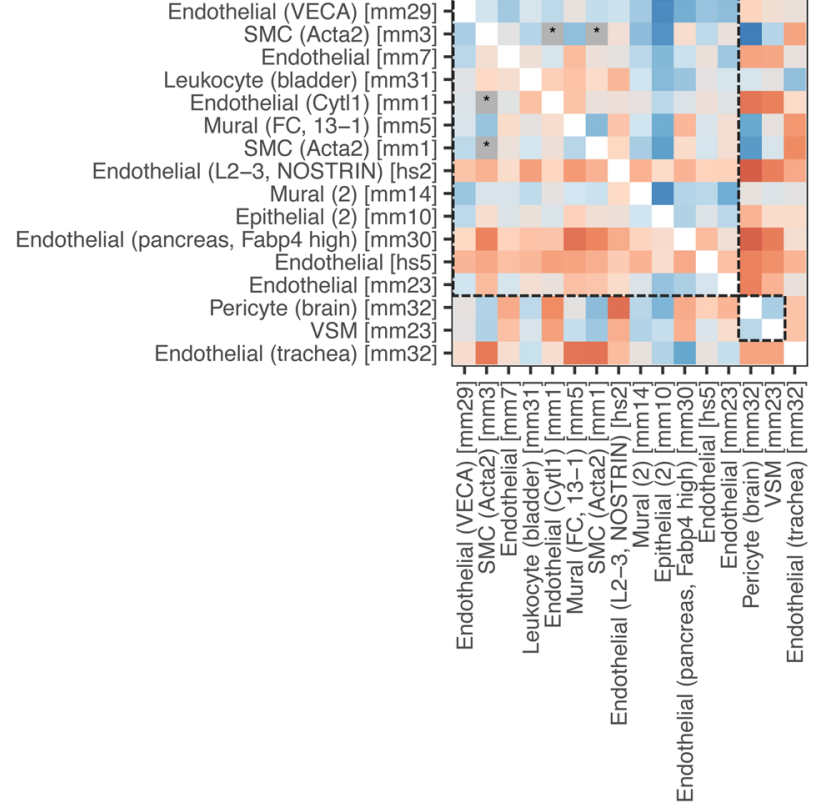

C

b

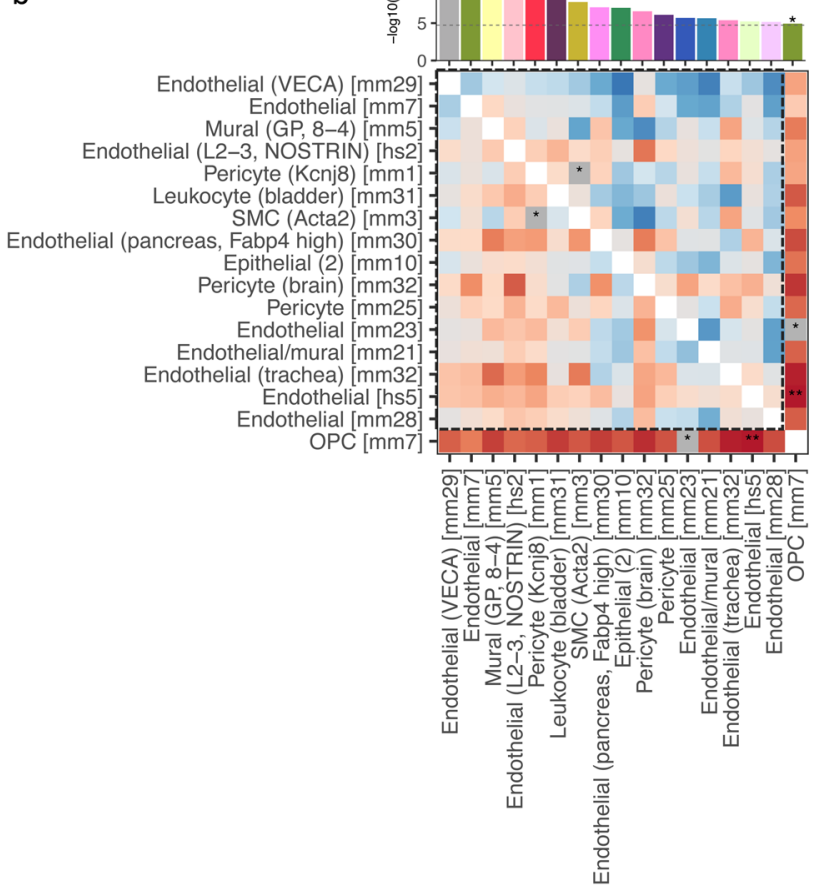

d
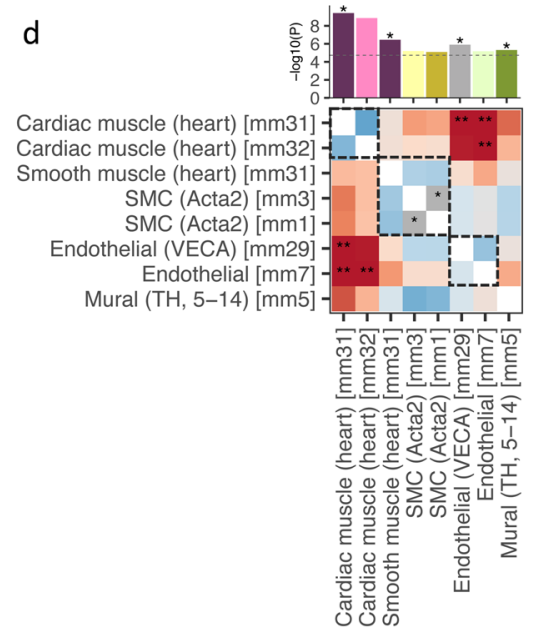
Endothelial [mm7] Mural (GP, 8-4) [mm5 Endothelial (L2-3, NOSTRIN) [hs2] SMC (Acta2) [mm3] Leukocyte (bladder) [mm31] Epithelial (2) [mm10] ericyte (Kcnj8) [mm1] Mural (2) [mm14] Endothelial [mm28] helial/mural [mm21] Endothelial [hs10] Endothelial (trachea) [mm32 Endothelial [mm23] VSM [mm23] Pericyte [mm25] Pericyte (brain) [mm32] Oligodendrocyte (2) [mm7]

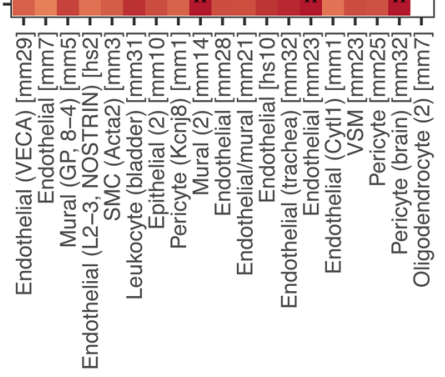

[hs1] Allen_hs_LGN [hs2] Allen_hs_MTG

[hs3] DroNc_hs [hs4] GSE104276 [hs5] GSE67835

[hs6] GSE81547

[hs7] GSE84133_hs

[hs 10 이 Linnarsson_GSE76381_hs [hs11] PBMC_10x_68k [mm1] Allen_mm_ALM2 [mm2] Allen_mm_LGd2 [mm3] Allen_mm_VISp2 [mm5] DropViz [mm7] GSE106678 [mm8] GSE82187 [mm9] GSE84133_mm [mm10] GSE87544 [mm14] GSE93374 [mm15] GSE98816 [mm17] Linnarsson_GSE101601_mm [mm19] Linnarsson_GSE104323 [mm20] Linnarsson_GSE59739 [mm21] Linnarsson_GSE6036 [mm22] Linnarsson_GSEG7602 [mm23] Linnarsson_GSE74672
Proportional

[mm24] Linnarsson_GSE75330 [mm25] Linnarsson_GSE76381_mm [mm27] Linnarsson_GSE95315 [mm28] Linnarsson_GSE95752 [mm29] Linnarsson_MouseBrainAtlas [mm30] MouseCellitlas

[mm31] TabulaMuris_droplet [mm32] TabulaMuris_FACS significance

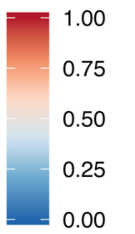



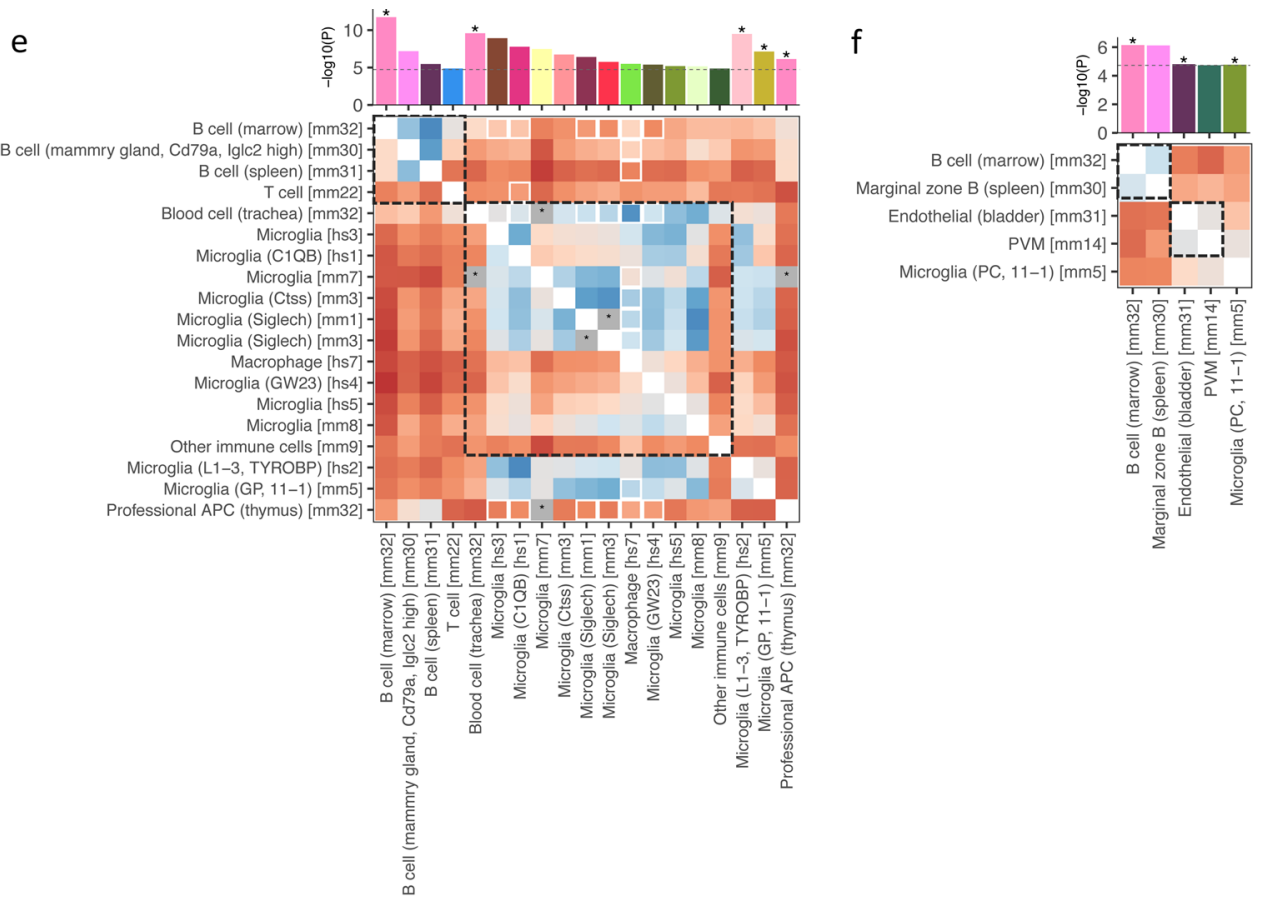

g

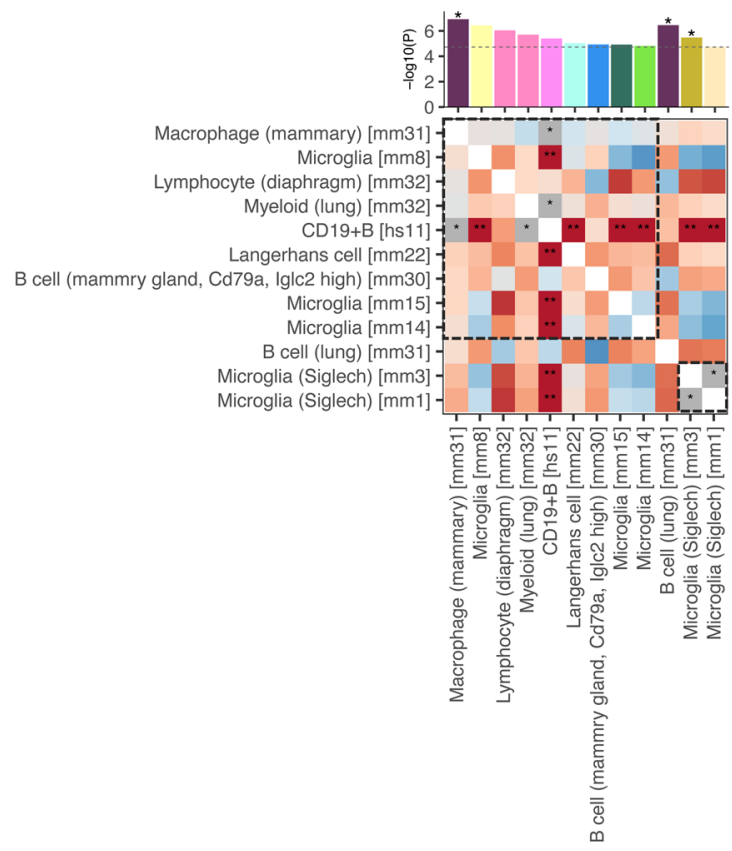

h
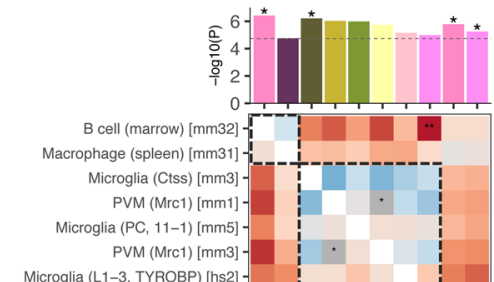

Microglia (L1-3, TYROBP) [hs 2] -

Macrophage (pancreas) [mm30] -

Professional APC (aorta) [mm32] Dendritic (spleen, siglech high) [mm30]

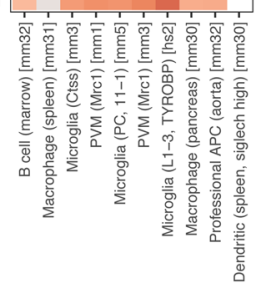

[hs1] Allen_hs_LGN

[mm1] Allen_mm_ALM2

[mm2] Allen_mm_LGd2

[mm3] Allen_mm_VISp2

[hs3] DroNc_hs

[hs4] GSE104276

[hs5] GSE67835

[hS6] GSE81547

[hs7] GSE84133_hs

[mm5] DropViz

[mm7] GSE106678

[mm8] GSE82187

[hs10] Linnarsson_GSE76381_hs

[mm9] GSE84133_mm

[mm10] GSE87544

[hs11] PBMC_10x_68k

[mm11] GSE89164

[mm14] GSE93374 [mm15] GSE98816 [mm17] Linnarsson_GSE101601_mm

[mm19] Linnarsson_GSE104323

[mm20] Linnarsson_GSE59739

[mm21] Linnarsson_GSE60361

[mm22] Linnarsson GSE67602

[mm23] Linnarsson_GSE74672
Proportional

[mm24] Linnarsson_GSE75330 [mm25] Linnarsson_GSE76381_mm

[mm27] Linnarsson_GSE95315 [mm28] Linnarsson_GSE95752 [mm29] Linnarsson_MouseBrainAtlas [mm30] MouseCellitlas

[mm31] TabulaMuris_droplet

[mm32] TabulaMuris_FACS significance

$=1.00$
0.75
0.50
$=0.25$
$-\quad 0.00$


i

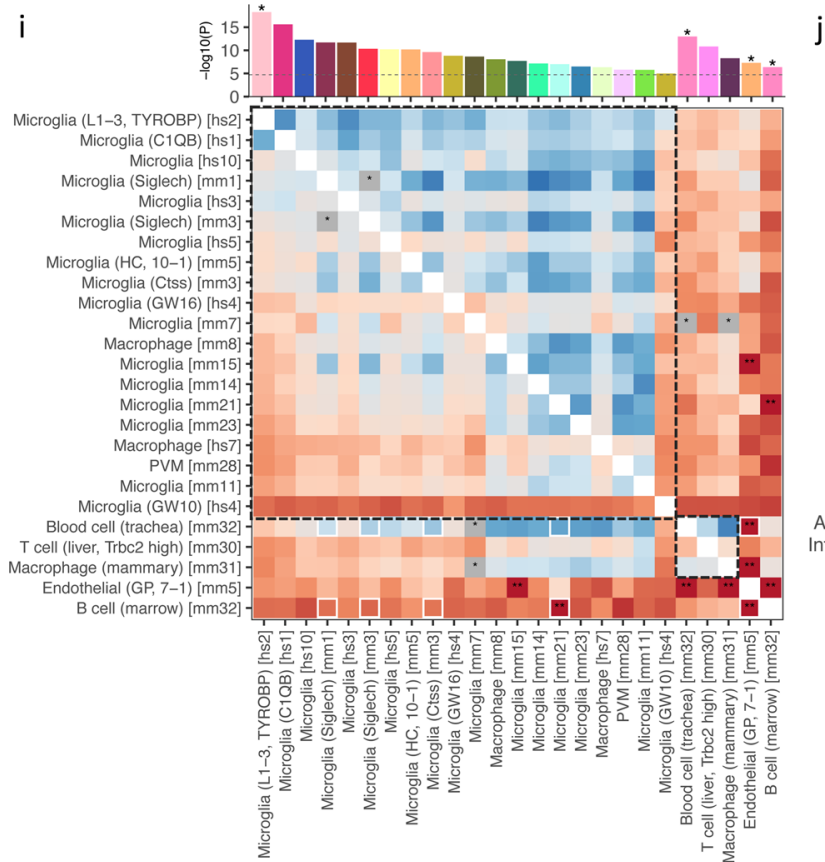

k

[hs1] Allen_hs_LGN

[hs2] Allen_hs_MTG

[hs3] DroNc_hs

[hS4] GSE104276

[hs5] GSE67835

[hs6] GSE81547

[hs7] GSE84133_hs

[hs10] Linnarsson_GSE76381_hs

[hs 11] PBMC_10x_68k

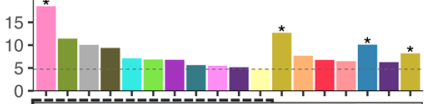

Neuron [mm32]

Neuron [mm8] GABAergic (GW26) [hs4]

idal neuron (CA3) [mm19] layer 4 (Plcxd2) [mm17] neuron (CA1) [mm21] Neuron [mm30 Neuron [hs5] , 7-1) [mm5] p layer pyramidal cells layer 5 ( $\mathrm{FC}, 7-1)$ [mm5] Excitatory neuron (Neurod6) [mm2] GABAergic [hs10]
Gat GABAergic [hs10] Lateral neuroblast [mm25]
Globus pallidus externus (GP, 2-17) [mm5]

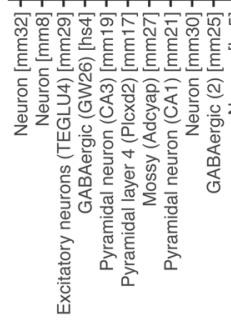

1

[mm3] Allen_mm_VISp2

[mm5] DropViz

[mm7] GSE106678

[mm8] GSE82187

[mm9] GSE84133_mm

[mm10] GSE87544
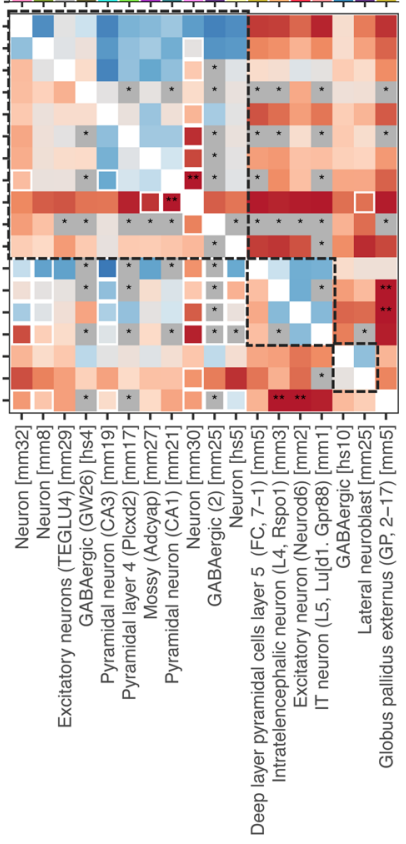

।

\section{।}

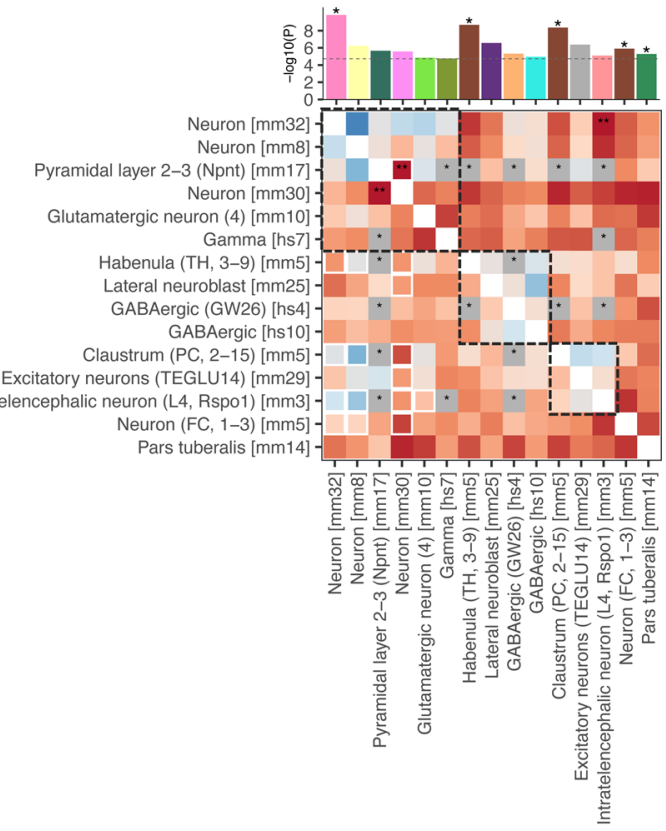

Proportional

significance

[mm24] Linnarsson_GSE75330

[mm25] Linnarsson_GSE76381_mm

[mm27] Linnarsson_GSE95315

[mm28] Linnarsson_GSE95752 [mm15] GSE98816

[mm17] Linnarsson_GSE101601_mm

[mm19] Linnarsson_GSE104323

[mm20] Linnarsson_GSE59739

[mm21] Linnarsson_GSE6036

[mm22] Linnarsson GSE67602

[mm23] Linnarsson_GSE74672 

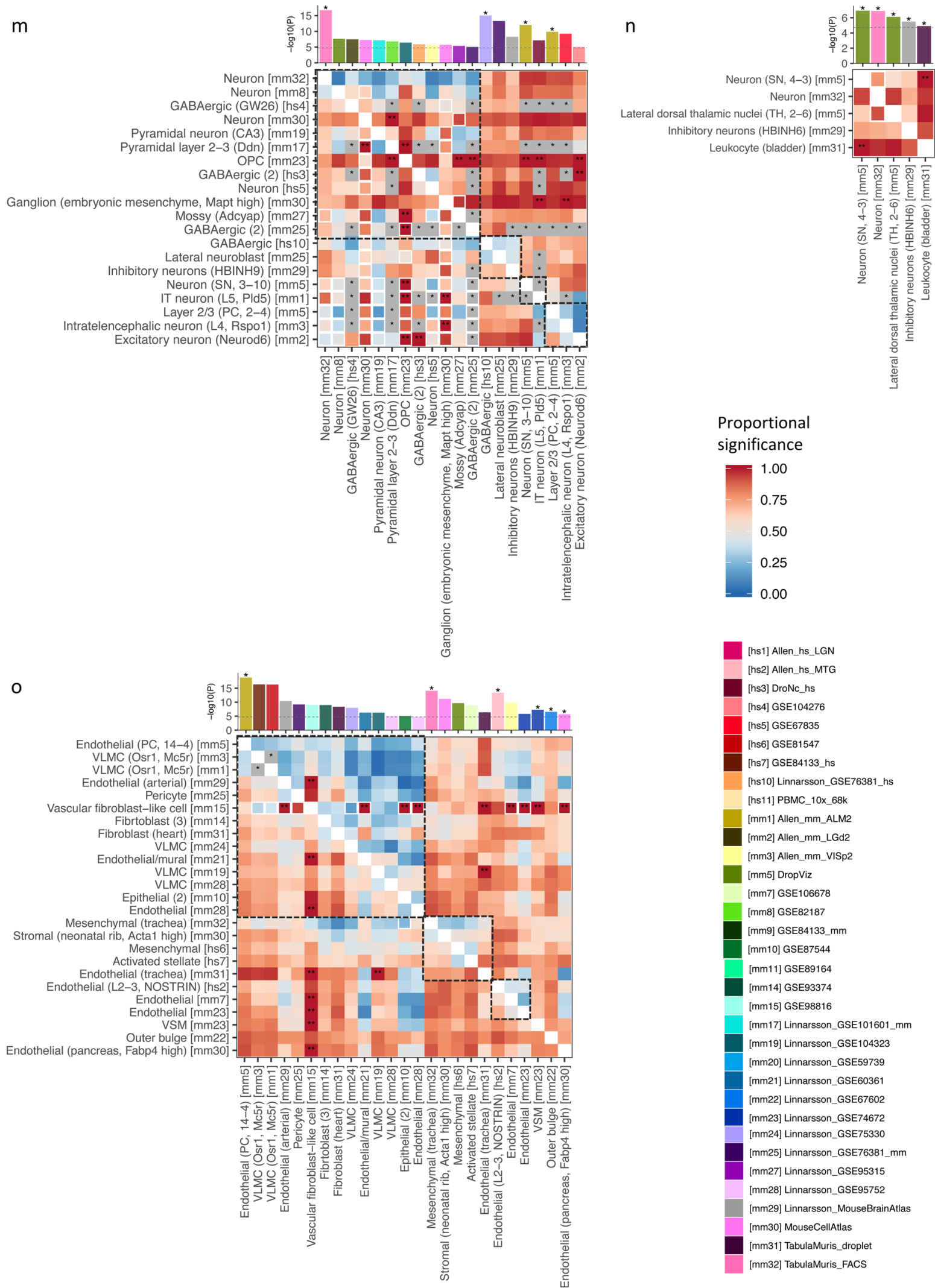

[hs1] Allen_hs_LGN [hs2] Allen_hs_MTG

[hs3] DroNc_hs [hs4] GSE104276

[hs5] GSE67835

[hs6] GSE81547

[hs7] GSE84133 hs [hs10] Linnarsson_GSE76381_hs [hs11] PBMC_10x_68k [mm1] Allen_mm_ALM2 [mm2] Allen_mm_LGd2 [mm3] Allen_mm_VISp2 [mm5] DropViz [mm7] GSE106678 [mm8] GSE82187 [mm9] GSE84133_mm [mm10] GSE87544 [mm11] GSE89164 [mm14] GSE93374 [mm15] GSE98816 [mm17] Linnarsson_GSE101601_mm [mm19] Linnarsson_GSE104323 [mm20] Linnarsson_GSE59739 [mm21] Linnarsson_GSE60361 [mm22] Linnarsson_GSE67602 [mm23] Linnarsson GSE74672 [mm24] Linnarsson_GSE75330 [mm25] Linnarsson_GSE76381_mm [mm27] Linnarsson_GSE95315 [mm28] Linnarsson_GSE95752 [mm29] Linnarsson_MouseBrainAtlas [mm30] MouseCellAtlas [mm31] TabulaMuris_droplet [mm32] TabulaMuris_FACS

The HTML has been updated to include a corrected version of the Supplementary Information. 
(c) Open Access This article is licensed under a Creative Commons Attribution 4.0 International License, which permits use, sharing, adaptation, distribution and reproduction in any medium or format, as long as you give appropriate credit to the original author(s) and the source, provide a link to the Creative Commons license, and indicate if changes were made. The images or other third party material in this article are included in the article's Creative Commons license, unless indicated otherwise in a credit line to the material. If material is not included in the article's Creative Commons license and your intended use is not permitted by statutory regulation or exceeds the permitted use, you will need to obtain permission directly from the copyright holder. To view a copy of this license, visit http://creativecommons.org/licenses/by/4.0/.

(C) The Author(s) 2020 